IJMMS 29:2 (2002) 63-70

PII. S016117120201195X

http://ijmms.hindawi.com

(c) Hindawi Publishing Corp.

\title{
FUZZY IMPLICATIVE HYPER BCK-IDEALS OF HYPER BCK-ALGEBRAS
}

\author{
YOUNG BAE JUN and WOOK HWAN SHIM
}

\author{
Received 8 February 2001
}

\begin{abstract}
We consider the fuzzification of the notion of implicative hyper BCK-ideals, and then investigate several properties. Using the concept of level subsets, we give a characterization of a fuzzy implicative hyper BCK-ideal. We state a relation between a fuzzy hyper BCK-ideal and a fuzzy implicative hyper BCK-ideal. We establish a condition for a fuzzy hyper BCK-ideal to be a fuzzy implicative hyper BCK-ideal. Finally, we introduce the notion of hyper homomorphisms of hyper BCK-algebras, and discuss related properties.
\end{abstract}

2000 Mathematics Subject Classification: 06F35, 03G25, 20N20, 03B52.

1. Introduction. The hyper structure theory (called also multialgebras) is introduced in 1934 by Marty [5] at the 8th Congress of Scandinavian Mathematicians. Around the 40's, several authors worked on hypergroups, especially in France and in the United States, but also in Italy, Russia, and Japan. Over the following decades, many important results appeared, but above all since the 70's onwards the most luxuriant flourishing of hyper structures has been seen. Hyper structures have many applications to several sectors of both pure and applied sciences. Jun et al. [4] applied the hyper structures to BCK-algebras, and introduced the concept of a hyper BCK-algebra which is a generalization of a BCK-algebra, and investigated some related properties. They also introduced the notion of a hyper BCK-ideal and a weak hyper BCK-ideal, and gave relations between hyper BCK-ideals and weak hyper BCK-ideals. Jun and Xin [1] considered the fuzzification of the notion of a (weak, strong, reflexive) hyper BCK-ideal, and investigated the relations among them. In this paper, we deal with the fuzzification of the notion of implicative hyper BCK-ideals. We show that every fuzzy implicative hyper BCK-ideal is a fuzzy hyper BCK-ideal, but not converse. We provide a condition for a fuzzy hyper BCK-ideal to be a fuzzy implicative hyper BCK-ideal. Using the notion of level subsets of a fuzzy set, we give a characterization of a fuzzy implicative hyper BCK-ideal. We introduce the concept of hyper homomorphisms of hyper BCK-algebras, and we show that the hyper homomorphic preimage of a fuzzy implicative hyper BCK-ideal is also a fuzzy implicative hyper BCK-ideal.

2. Preliminaries. We include some elementary aspects of hyper BCK-algebras that are necessary for this paper, and for more details we refer to [2, 4].

Let $H$ be a nonempty set endowed with a hyper operation "०", that is, $\circ$ is a function from $H \times H$ to $\mathscr{P} *(H)=\mathscr{P}(H) \backslash\{\varnothing\}$. For two subsets $A$ and $B$ of $H$, denote by $A \circ B$ the set $\bigcup_{a \in A, b \in B} a \circ b$. 
By a hyper BCK-algebra we mean a nonempty set $H$ endowed with a hyper operation "。" and a constant 0 satisfying the following axioms:

$(\mathrm{HK} 1)(x \circ z) \circ(y \circ z) \ll x \circ y$,

(HK2) $(x \circ y) \circ z=(x \circ z) \circ y$,

(HK3) $x \circ H \ll\{x\}$,

(HK4) $x \ll y$ and $y \ll x$ imply $x=y$,

for all $x, y, z \in H$, where $x \ll y$ is defined by $0 \in x \circ y$ and for every $A, B \subseteq H, A \ll B$ is defined by for all $a \in A$, there exists $b \in B$ such that $a \ll b$.

In any hyper BCK-algebra $H$, the following hold (see [2, 4]):

(P1) $A \subseteq B$ implies $A \ll B$,

(P2) $0 \circ x=\{0\}$,

(P3) $(A \circ B) \circ C=(A \circ C) \circ B, A \circ B \ll A$, and $0 \circ A \ll\{0\}$,

(P4) $x \circ 0=\{x\}$ and $A \circ 0=A$

for all $x \in H$ and for all nonempty subsets $A, B$, and $C$ of $H$.

Proposition 2.1 (see Jun et al. [4, Proposition 3.3]). In a hyper BCK-algebra $H$, the condition (HK3) is equivalent to the condition

(P5) $x \circ y \ll\{x\}$ for all $x, y \in H$.

Definition 2.2 (see Jun et al. [4, Definition 3.14]). A nonempty subset $I$ of a hyper BCK-algebra $H$ is called a hyper BCK-ideal of $H$ if

(HI1) $0 \in I$,

(HI2) $x \circ y \ll I$ and $y \in I$ imply $x \in I$

for all $x, y \in H$.

By a fuzzy set $\mu$ in $H$ we mean a function $\mu: H \rightarrow[0,1]$. For a fuzzy set $\mu$ in $H$ and $t \in[0,1]$, the set $U(\mu ; t):=\{x \in H \mid \mu(x) \geq t\}$ is called a level subset of $\mu$.

DeFinItion 2.3 (see Jun et al. [1, Definition 3.1]). A fuzzy set $\mu$ in a hyper BCKalgebra $H$ is called a fuzzy hyper $B C K$-ideal of $H$ if

(FHI1) $x \ll y$ implies $\mu(y) \leq \mu(x)$,

(FHI2) $\mu(x) \geq \min \left\{\inf _{a \in x \circ y} \mu(a), \mu(y)\right\}$

for all $x, y \in H$.

Theorem 2.4 (see Jun et al. [1, Theorem 3.17]). Let $\mu$ be a fuzzy set in a hyper $B C K$-algebra $H$. Then $\mu$ is a fuzzy hyper BCK-ideal of $H$ if and only if $U(\mu ; t)$ is a hyper $B C K$-ideal of $H$ whenever $U(\mu ; t) \neq \varnothing$ for $t \in[0,1]$.

3. Fuzzy implicative hyper BCK-ideals. In what follows let $H$ denote a hyper BCKalgebra unless otherwise specified.

DeFinITION 3.1 (see Jun et al. [3]). A nonempty subset $I$ of $H$ is called an implicative hyper BCK-ideal of $H$ if it satisfies (HI1) and

(HI3) $(x \circ z) \circ(y \circ x) \ll I$ and $z \in I$ imply $x \in I$

for all $x, y, z \in H$.

LEMMA 3.2 (see Jun et al. [3, Proposition 3.6]). Let I be a hyper BCK-ideal of H, then $I$ is implicative if and only if $x \circ(y \circ x) \ll I$ implies $x \in I$. 
DeFinITION 3.3. A fuzzy set $\mu$ in $H$ is called a fuzzy implicative hyper BCK-ideal of $H$ if it satisfies (FHI1) and

(FHI3) $\mu(x) \geq \min \left\{\inf _{w \in(x \circ z) \circ(y \circ x)} \mu(w), \mu(z)\right\}$ for all $x, y, z \in H$.

EXAMPLE 3.4. Consider a hyper BCK-algebra $H=\{0, a, b\}$ with the following Cayley table:

\begin{tabular}{c|ccc}
\hline$\circ$ & 0 & $a$ & $b$ \\
\hline 0 & $\{0\}$ & $\{0\}$ & $\{0\}$ \\
$a$ & $\{a\}$ & $\{0\}$ & $\{0\}$ \\
$b$ & $\{b\}$ & $\{a, b\}$ & $\{0, a, b\}$ \\
\hline
\end{tabular}

Define a fuzzy set $\mu$ in $H$ by $\mu(0)=\mu(a)=0.7$ and $\mu(b)=0.2$. It is routine to verify that $\mu$ is a fuzzy implicative hyper BCK-ideal of $H$.

THEOREM 3.5. Let $\mu$ be a fuzzy set in $H$. Then $\mu$ is a fuzzy implicative hyper BCKideal of $H$ if and only if $U(\mu ; t)$ is an implicative hyper BCK-ideal of $H$ whenever $U(\mu ; t) \neq \varnothing$ for $t \in[0,1]$.

Proof. Let $\mu$ be a fuzzy implicative hyper BCK-ideal of $H$ and assume that $U(\mu ; t) \neq$ $\varnothing$ for $t \in[0,1]$. Let $x, y, z \in H$ be such that $(x \circ z) \circ(y \circ x) \ll U(\mu ; t)$ and $z \in U(\mu ; t)$. For any $w \in(x \circ z) \circ(y \circ x)$, there exists $a \in U(\mu ; t)$ such that $w \ll a$. It follows from (FHI1) that $\mu(w) \geq \mu(a) \geq t$. Hence

$$
\mu(x) \geq \min \left\{\inf _{w \in(x \circ z) \circ(y \circ x)} \mu(w), \mu(z)\right\} \geq \min \{t, \mu(z)\} \geq t,
$$

and so $x \in U(\mu ; t)$. Thus $U(\mu ; t)$ is an implicative hyper BCK-ideal of $H$. Conversely, suppose that for each $t \in[0,1], U(\mu ; t)(\neq \varnothing)$ is an implicative hyper BCK-ideal of $H$. For any $x, y, z \in H$, let

$$
t=\min \left\{\inf _{w \in(x \circ z) \circ(y \circ x)} \mu(w), \mu(z)\right\} .
$$

Then $z \in U(\mu ; t)$ and for every $a \in(x \circ z) \circ(y \circ x)$, we have

$$
\mu(a) \geq \inf _{w \in(x \circ z) \circ(y \circ x)} \mu(w) \geq \min \left\{\inf _{w \in(x \circ z) \circ(y \circ x)} \mu(w), \mu(z)\right\}=t .
$$

Thus $a \in U(\mu ; t)$, and so $(x \circ z) \circ(y \circ x) \subseteq U(\mu ; t)$. Hence $(x \circ z) \circ(y \circ x) \ll U(\mu ; t)$ by (P1). Combining $z \in U(\mu ; t)$ and $U(\mu ; t)$ being an implicative hyper BCK-ideal of $H$, we get $x \in U(\mu ; t)$ and thus

$$
\mu(x) \geq t=\min \left\{\inf _{w \in(x \circ z) \circ(y \circ x)} \mu(w), \mu(z)\right\} .
$$

Therefore $\mu$ is a fuzzy implicative hyper BCK-ideal of $H$.

THEOREM 3.6. For any subset I of $H$, let $\mu_{I}$ be a fuzzy set in $H$ defined by

$$
\mu_{I}(x):= \begin{cases}t_{1} & \text { if } x \in I, \\ t_{2} & \text { otherwise }\end{cases}
$$

for all $x \in H$, where $t_{1}, t_{2} \in[0,1]$ with $t_{1}>t_{2}$. Then $I$ is an implicative hyper BCK-ideal of $H$ if and only if $\mu_{I}$ is a fuzzy implicative hyper BCK-ideal of $H$. 
Proof. Assume that $I$ is an implicative hyper BCK-ideal of $H$. Note that

$$
U\left(\mu_{I} ; t\right)= \begin{cases}\varnothing & \text { if } t_{1}<t \leq 1, \\ I & \text { if } t_{2}<t \leq t_{1}, \\ H & \text { if } 0 \leq t \leq t_{2},\end{cases}
$$

which is an implicative hyper BCK-ideal of $H$ for all $t \in[0,1]$. It follows from Theorem 3.5 that $\mu_{I}$ is a fuzzy implicative hyper BCK-ideal of $H$. Conversely, if $\mu_{I}$ is a fuzzy implicative hyper BCK-ideal of $H$, then $U\left(\mu_{I} ; t_{1}\right)=I$ is an implicative hyper BCK-ideal of $H$.

THEOREM 3.7. Every fuzzy implicative hyper BCK-ideal is a fuzzy hyper BCK-ideal.

Proof. Let $\mu$ be a fuzzy implicative hyper BCK-ideal of $H$. Then

$$
\begin{aligned}
\mu(x) & \geq \min \left\{\inf _{w \in(x \circ y) \circ(0 \circ x)} \mu(w), \mu(y)\right\} \\
& =\min \left\{\inf _{w \in(x \circ y) \circ 0} \mu(w), \mu(y)\right\} \\
& =\min \left\{\inf _{w \in x \circ y} \mu(w), \mu(y)\right\}
\end{aligned}
$$

for all $x, y \in H$. Hence $\mu$ is a fuzzy hyper BCK-ideal of $H$.

The converse of Theorem 3.7 may not be true as seen in the following example.

EXAMPLE 3.8. Consider a hyper BCK-algebra $H=\{0, a, b\}$ with the following Cayley table:

\begin{tabular}{c|ccc}
\hline$\circ$ & 0 & $a$ & $b$ \\
\hline 0 & $\{0\}$ & $\{0\}$ & $\{0\}$ \\
$a$ & $\{a\}$ & $\{0\}$ & $\{0\}$ \\
$b$ & $\{b\}$ & $\{a\}$ & $\{0, a\}$ \\
\hline
\end{tabular}

Define a fuzzy set $\mu$ in $H$ by $\mu(0)=t_{1}$ and $\mu(a)=\mu(b)=t_{2}$ for every $t_{1}>t_{2}$ in $[0,1]$. It can be easily checked that $\mu$ is a fuzzy hyper BCK-ideal of $H$, but $\mu$ is not a fuzzy implicative hyper BCK-ideal of $H$ since

$$
\mu(a)=t_{2}<t_{1}=\min \left\{\inf _{w \in(a \circ 0) \circ(b \circ a)} \mu(w), \mu(0)\right\} .
$$

We give a condition for a fuzzy hyper BCK-ideal to be a fuzzy implicative hyper BCK-ideal.

THEOREM 3.9. Let $\mu$ be a fuzzy hyper BCK-ideal of $H$. Then $\mu$ is a fuzzy implicative hyper BCK-ideal of $H$ if and only if $\mu(x) \geq \inf _{w \in x \circ(y \circ x)} \mu(w)$ for all $x, y \in H$.

Proof. Assume that $\mu$ is a fuzzy implicative hyper BCK-ideal of $H$. Then

$$
\mu(x) \geq \min \left\{\inf _{w \in(x \circ 0) \circ(y \circ x)} \mu(w), \mu(0)\right\}=\inf _{w \in x \circ(y \circ x)} \mu(w)
$$

for all $x, y \in H$. Conversely, let $\mu$ be a fuzzy hyper BCK-ideal of $H$ that satisfies 
the inequality $\mu(x) \geq \inf _{w \in x \circ(y \circ x)} \mu(w)$ for all $x, y \in H$. It is sufficient to show that $U(\mu ; t)(\neq \varnothing)$ is an implicative hyper BCK-ideal of $H$. Let $x, y, z \in H$ be such that $(x \circ z) \circ(y \circ x) \ll U(\mu ; t)$ and $z \in U(\mu ; t)$. Then $\mu(z) \geq t$ and $(x \circ(y \circ x)) \circ z \ll U(\mu ; t)$, and so $w \circ z \ll U(\mu ; t)$ for all $w \in x \circ(y \circ x)$. Since $U(\mu ; t)$ is a hyper BCK-ideal of $H$ (see Theorem 2.4), it follows that $w \in U(\mu ; t)$, that is, $x \circ(y \circ x) \subseteq U(\mu ; t)$. Hence

$$
\mu(x) \geq \inf _{w \in x \circ(y \circ x)} \mu(w) \geq \inf _{w \in U(\mu ; t)} \mu(w) \geq t,
$$

which implies that $x \in U(\mu ; t)$. Therefore, $U(\mu ; t)$ is an implicative hyper BCK-ideal of $H$, and so $\mu$ is a fuzzy implicative hyper BCK-ideal of $H$.

THEOREM 3.10. If $\mu$ is a fuzzy implicative hyper BCK-ideal of $H$, then the set

$$
I:=\{x \in H \mid \mu(x)=\mu(0)\}
$$

is an implicative hyper BCK-ideal of $H$.

Proof. Clearly $0 \in I$. Let $x, y \in H$ be such that $x \circ y \ll I$ and $y \in I$. Then $\mu(y)=$ $\mu(0)$, and for each $a \in x \circ y$ there exists $z \in I$ such that $a \ll z$. Thus $\mu(a) \geq \mu(z)=$ $\mu(0)$ by (FHI1), and so $\mu(a)=\mu(0)$. It follows from (FHI2) that

$$
\mu(x) \geq \min \left\{\inf _{a \in x \circ y} \mu(a), \mu(y)\right\}=\mu(0),
$$

so that $\mu(x)=\mu(0)$. Hence $x \in I$, which shows that $I$ is a hyper BCK-ideal of $H$. Now let $x, y \in H$ be such that $x \circ(y \circ x) \ll I$. Then for every $b \in x \circ(y \circ x)$ there exists $w \in I$ such that $b \ll w$. Thus $\mu(b) \geq \mu(w)=\mu(0)$ and so $\mu(b)=\mu(0)$. Using Theorem 3.9, we have

$$
\mu(x) \geq \inf _{b \in x \circ(y \circ x)} \mu(b)=\mu(0),
$$

and thus $\mu(x)=\mu(0)$, that is, $x \in I$. Therefore, by means of Lemma 3.2, $I$ is an implicative hyper BCK-ideal of $H$.

DeFINITION 3.11. A mapping $f: G \rightarrow H$ of hyper BCK-algebras is called a hyper homomorphism if

(HH1) $f(0)=0$,

(HH2) $f(x \circ y)=f(x) \circ f(y)$ for all $x, y \in G$.

Proposition 3.12. Let $f: G \rightarrow H$ be a hyper homomorphism of hyper BCK-algebras. If $x \ll y$ in $G$, then $f(x) \ll f(y)$ in $H$.

Proof. Let $x, y \in G$ be such that $x \ll y$. Then $0 \in x \circ y$, and so $0=f(0) \in$ $f(x \circ y)=f(x) \circ f(y)$. Therefore, $f(x) \ll f(y)$ in $H$.

THEOREM 3.13. Let $f: G \rightarrow H$ be a hyper homomorphism of hyper BCK-algebras. If $I$ is an implicative hyper BCK-ideal of $H$, then $f^{-1}(I)$ is an implicative hyper BCK-ideal of $G$.

Proof. Clearly $0 \in f^{-1}(I)$. Let $x, y \in G$ be such that $x \circ y \ll f^{-1}(I)$ and $y \in$ $f^{-1}(I)$. Then $f(y) \in I$, and for every $z \in x \circ y$ there exists $w \in f^{-1}(I)$ such that 
$z \ll w$, that is, $0 \in z \circ w$. It follows that

$$
0=f(0) \in f(z \circ w)=f(z) \circ f(w) \subseteq f(x \circ y) \circ I=(f(x) \circ f(y)) \circ I
$$

so that $f(x) \circ f(y) \ll I$. Since $I$ is a hyper BCK-ideal of $H$, we have $f(x) \in I$, that is, $x \in f^{-1}(I)$ by (HI2). Hence $f^{-1}(I)$ is a hyper BCK-ideal of $G$. Now let $x, y \in G$ be such that $x \circ(y \circ x) \ll f^{-1}(I)$. Then for each $a \in x \circ(y \circ x)$, there exists $b \in f^{-1}(I)$ such that $a \ll b$, that is, $0 \in a \circ b$. Thus

$$
\begin{aligned}
0 & =f(0) \in f(a \circ b)=f(a) \circ f(b) \subseteq f(x \circ(y \circ x)) \circ I \\
& =(f(x) \circ(f(y) \circ f(x))) \circ I,
\end{aligned}
$$

and so $f(x) \circ(f(y) \circ f(x)) \ll I$. Since $I$ is implicative, it follows from Lemma 3.2 that $f(x) \in I$ so that $x \in f^{-1}(I)$. This shows that $f^{-1}(I)$ is an implicative hyper BCK-ideal of $G$.

LEMma 3.14 (see Jun et al. [2, Proposition 3.7]). Let A be a subset of H. If I is a hyper $B C K$-ideal of $H$ such that $A \ll I$, then $A$ is contained in $I$.

THEOREM 3.15. If $f: G \rightarrow H$ is a hyper homomorphism of hyper BCK-algebras, then $\operatorname{ker}(f):=\{x \in G \mid f(x)=0\}$, called the kernel of $f$, is an implicative hyper BCK-ideal of $G$.

Proof. Clearly $0 \in \operatorname{ker}(f)$. Let $x, y \in G$ be such that $x \circ y \ll \operatorname{ker}(f)$ and $y \in$ $\operatorname{ker}(f)$. Then $f(y)=0$, and for each $a \in x \circ y$ there exists $b \in \operatorname{ker}(f)$ such that $a \ll b$. It follows from (HH2) and (P4) that $a \in \operatorname{ker}(f)$ so that

$$
0=f(a) \in f(x \circ y)=f(x) \circ f(y)=f(x) \circ 0=f(x),
$$

that is, $x \in \operatorname{ker}(f)$. Hence $\operatorname{ker}(f)$ is a hyper BCK-ideal of $G$. Let $x, y \in G$ be such that $x \circ(y \circ x) \ll \operatorname{ker}(f)$. Then $x \circ(y \circ x) \subseteq \operatorname{ker}(f)$ by Lemma 3.14. Taking $y=0$ and using (P2) and (P4), we have $\{x\} \subseteq \operatorname{ker}(f)$, that is, $x \in \operatorname{ker}(f)$. It follows from Lemma 3.2 that $\operatorname{ker}(f)$ is an implicative hyper BCK-ideal of $G$.

Using Theorems 3.6 and 3.15, we know that if $f: G \rightarrow H$ is a hyper homomorphism of hyper BCK-algebras, then $\mu_{\operatorname{ker}(f)}$ is a fuzzy implicative hyper BCK-ideal of $G$.

THEOREM 3.16. Let $f: G \rightarrow H$ be an onto hyper homomorphism of hyper BCKalgebras. If I is an implicative hyper BCK-ideal of $G$ containing $\operatorname{ker}(f)$, then $f(I)$ is an implicative hyper BCK-ideal of $H$.

Proof. Note that $0=f(0) \in f(I)$. Let $x, y \in H$ be such that $x \circ y \ll f(I)$ and $y \in f(I)$. Since $f$ is onto, there exist $a, b \in G$ such that $f(a)=x$ and $f(b)=y$. Thus

$$
f(a \circ b)=f(a) \circ f(b)=x \circ y \ll f(I) .
$$

Let $w \in a \circ b$. Then $f(w) \ll f(z)$, that is, $0 \in f(w) \circ f(z)=f(w \circ z)$ for some $z \in I$. It follows that $w \circ z \subseteq \operatorname{ker}(f) \subseteq I$ so that $w \circ z \ll I$ by (P1). Since $I$ is a hyper BCK-ideal of $G$, we have $w \in I$ by (HI2). Hence $a \circ b \subseteq I$ and so $a \circ b \ll I$. Since $b \in I$, it follows 
from (HI2) that $a \in I$ so that $x=f(a) \in f(I)$. Hence $f(I)$ is a hyper BCK-ideal of $H$. Now let $x, y \in H$ be such that $x \circ(y \circ x) \ll f(I)$. Then

$$
f(a \circ(b \circ a))=f(a) \circ(f(b) \circ f(a))=x \circ(y \circ x) \ll f(I)
$$

for some $a, b \in G$. Let $u \in a \circ(b \circ a)$. Then there exists $c \in I$ such that $f(u) \ll f(c)$, that is, $0 \in f(u) \circ f(c)=f(u \circ c)$, which implies that $u \circ c \subseteq \operatorname{ker}(f) \subseteq I$. It follows from (P1) that $u \circ c \ll I$ so from (HI2) that $u \in I$. Thus $a \circ(b \circ a) \subseteq I$ and so $a \circ(b \circ a) \ll I$. Since $I$ is implicative, we get $a \in I$ by Lemma 3.2. Hence $x=f(a) \in f(I)$, and therefore $f(I)$ is implicative. This completes the proof.

THEOREM 3.17. Let $f: G \rightarrow H$ be an onto hyper homomorphism of hyper BCKalgebras. If $v$ is a fuzzy implicative hyper BCK-ideal of $H$, then the hyper homomorphic preimage $\mu$ of $\nu$ under $f$, that is, the fuzzy set $\mu$ in $G$ defined by $\mu(x)=\nu(f(x))$ for all $x \in G$, is a fuzzy implicative hyper BCK-ideal of $G$.

Proof. Let $x, y \in G$ be such that $x \ll y$. Then $f(x) \ll f(y)$ (see Proposition 3.12). Thus

$$
\mu(x)=v(f(x)) \geq v(f(y))=\mu(y),
$$

which proves (FHI1). For any $x \in G$, we have

$$
\mu(x)=v(f(x)) \geq \min \left\{\inf _{w \in f(x) \circ a} v(w), v(a)\right\}
$$

for all $a \in H$. Let $y$ be an arbitrary preimage of $a$ under $f$. Then

$$
w \in f(x) \circ a=f(x) \circ f(y)=f(x \circ y),
$$

which implies $w=f(b)$ for some $b \in x \circ y$. Hence

$$
\begin{aligned}
\mu(x) & \geq \min \left\{\inf _{f(b) \in f(x \circ y)} v(f(b)), v(f(y))\right\} \\
& =\min \left\{\inf _{b \in x \circ y} \mu(b), \mu(y)\right\} .
\end{aligned}
$$

Since $a$ is an arbitrary element of $H$, (3.22) is true for any $y \in G$. Therefore $\mu$ is a fuzzy hyper BCK-ideal of $G$. Since $v$ is a fuzzy implicative hyper BCK-ideal of $H$, it follows from Theorem 3.9 that

$$
\mu(x)=v(f(x)) \geq \inf _{w \in f(x) \circ(a \circ f(x))} v(w)
$$

for all $x \in G$ and $a \in H$. Let $y$ be an arbitrary element of $G$ such that $f(y)=a$. Then

$$
w \in f(x) \circ(a \circ f(x))=f(x) \circ(f(y) \circ f(x))=f(x \circ(y \circ x)),
$$

which implies that there exists $b \in x \circ(y \circ x)$ such that $f(b)=w$. Hence

$$
\mu(x) \geq \inf _{f(b) \in f(x \circ(y \circ x))} v(f(b))=\inf _{b \in x \circ(y \circ x)} \mu(b) .
$$

Since $a$ is arbitrary in $H$, (3.25) is also true for all $y \in G$. Using Theorem 3.9, we conclude that $\mu$ is a fuzzy implicative hyper BCK-ideal of $G$. 
ACKNOWLEDGEMENT. This work was supported by Korea Research Foundation Grant (KRF-99-005-D00003).

\section{REFERENCES}

[1] Y. B. Jun and X. L. Xin, Fuzzy hyper BCK-ideals of hyper BCK-algebras, to appear in Math. Japon.

[2] Scalar elements and hyper atoms of hyper BCK-algebras, Math. Japon. 2 (1999), no. 3, 303-309.

[3] _ Implicative hyper BCK-ideals of hyper BCK-algebras, Math. Japon. 52 (2000), no. 3, 435-443.

[4] Y. B. Jun, M. M. Zahedi, X. L. Xin, and R. A. Borrowed, On hyper BCK-algebras, Ital. J. Pure Appl. Math. (2000), no. 8, 127-136.

[5] F. Marty, Sur une generalization de la notion de groupe, 8th Congress Math. Scandinaves (Stockholm), 1934, pp. 45-49 (French).

Young Bae Jun: Department of Mathematics Education, Gyeongsang National UNIVERSITY, CHINJU 660-701, KOREA

E-mail address: ybjun@nongae.gsnu.ac.kr

Wook Hwan Shim: Department of Mathematics Education, GyeOngSang National UNIVERSITY, CHINJU 660-701, KOREA 


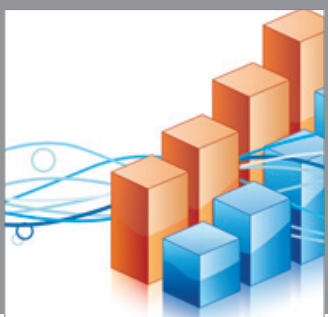

Advances in

Operations Research

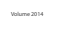

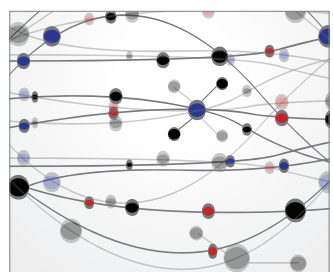

\section{The Scientific} World Journal
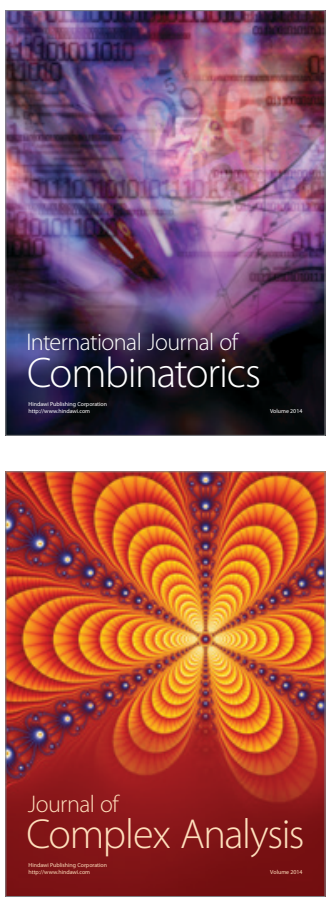

International Journal of

Mathematics and

Mathematical

Sciences
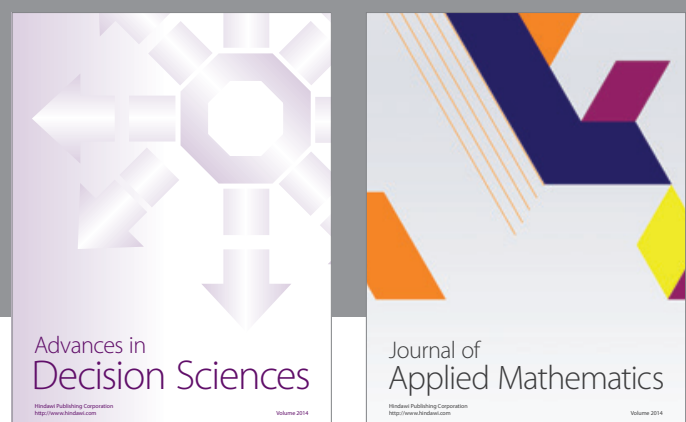

Journal of

Applied Mathematics
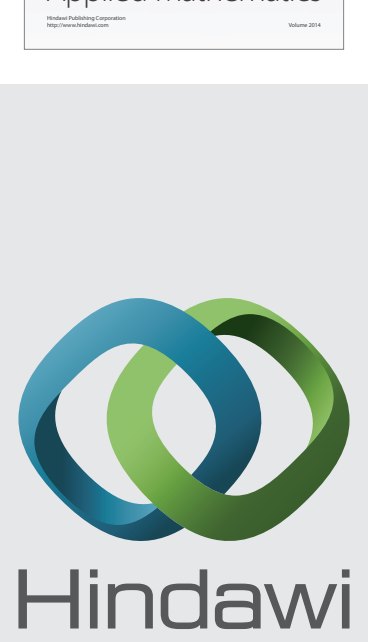

Submit your manuscripts at http://www.hindawi.com
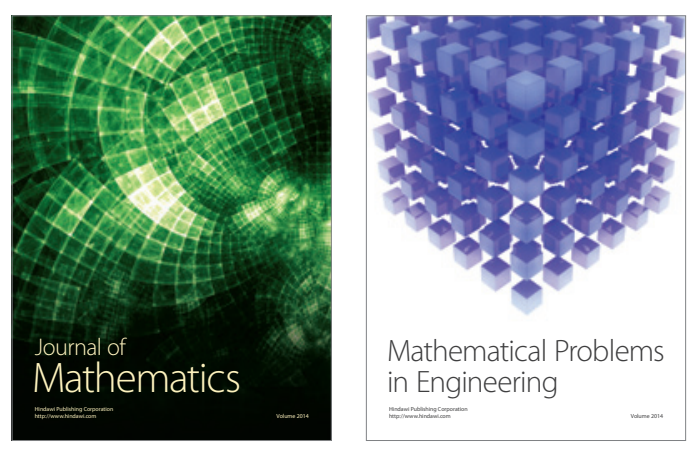

Mathematical Problems in Engineering
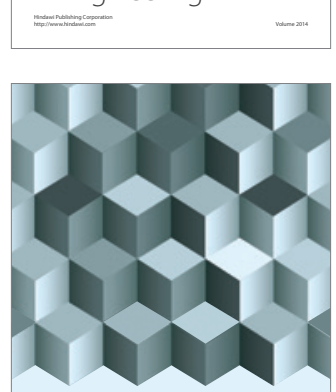

Journal of

Function Spaces
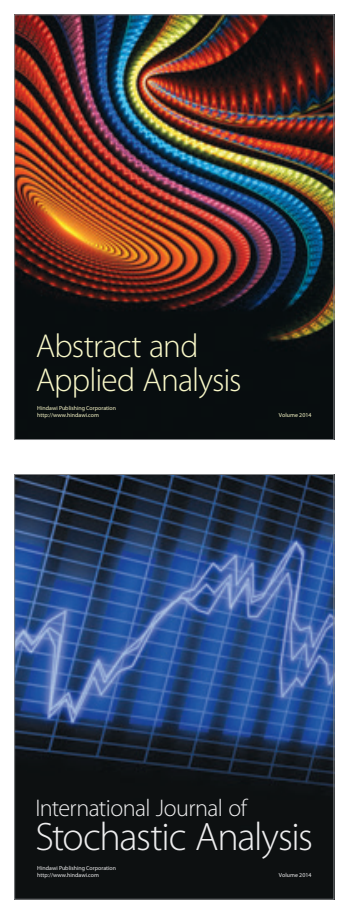

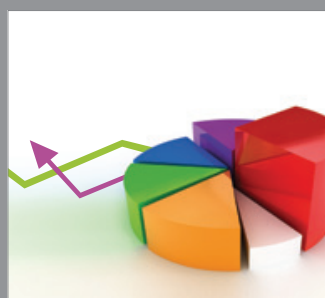

ournal of

Probability and Statistics

Promensencen
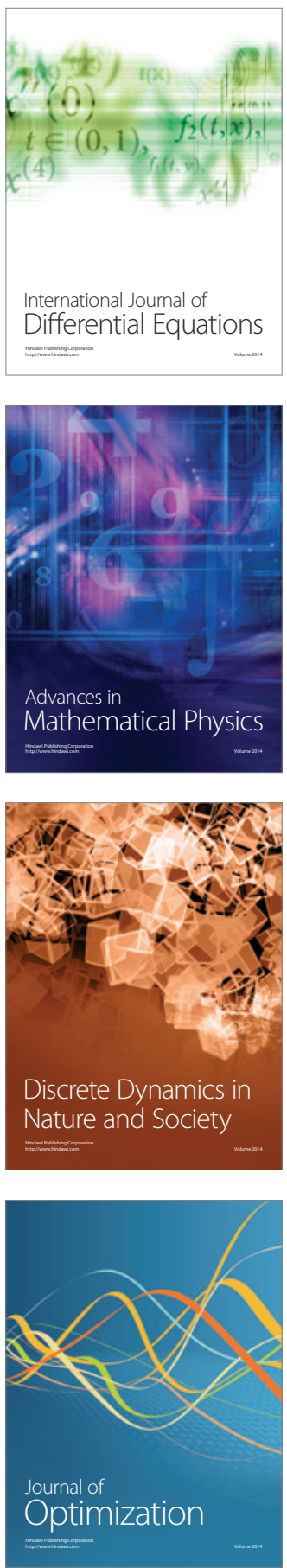